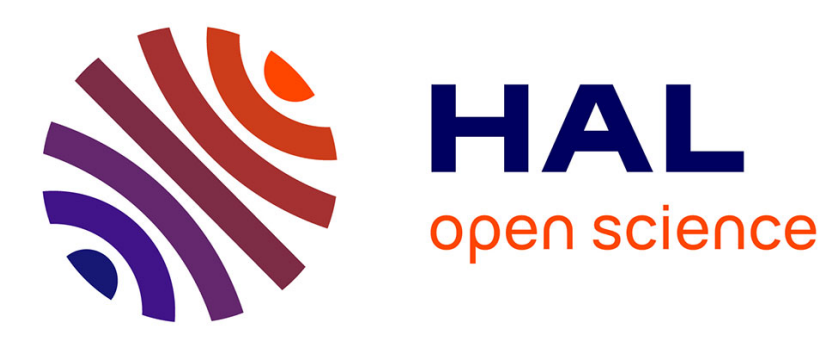

\title{
Des couvre-feux à Paris en 1958 et 1961 : Une mesure importée d'Algérie pour mieux lutter contre le FLN ?
}

Sylvie Thénault

\section{To cite this version:}

Sylvie Thénault. Des couvre-feux à Paris en 1958 et 1961 : Une mesure importée d'Algérie pour mieux lutter contre le FLN ?. Politix, 2008, nº 84 (4), pp.167. 10.3917/pox.084.0167 . hal-02355795

\section{HAL Id: hal-02355795 \\ https://hal.science/hal-02355795}

Submitted on 12 Nov 2019

HAL is a multi-disciplinary open access archive for the deposit and dissemination of scientific research documents, whether they are published or not. The documents may come from teaching and research institutions in France or abroad, or from public or private research centers.
L'archive ouverte pluridisciplinaire HAL, est destinée au dépôt et à la diffusion de documents scientifiques de niveau recherche, publiés ou non, émanant des établissements d'enseignement et de recherche français ou étrangers, des laboratoires publics ou privés. 


\title{
DES COUVRE-FEUX À PARIS EN 1958 ET 1961 : UNE MESURE IMPORTÉE D'ALGÉRIE POUR MIEUX LUTTER CONTRE LE FLN ?
}

\author{
Sylvie Thénault
}

De Boeck Supérieur | « Politix »

$2008 / 4 n^{\circ} 84 \mid$ pages 167 à 185

ISSN 0295-2319

ISBN 9782200924898

Article disponible en ligne à l'adresse :

https://www.cairn.info/revue-politix-2008-4-page-167.htm

Distribution électronique Cairn.info pour De Boeck Supérieur.

(C) De Boeck Supérieur. Tous droits réservés pour tous pays.

La reproduction ou représentation de cet article, notamment par photocopie, n'est autorisée que dans les limites des conditions générales d'utilisation du site ou, le cas échéant, des conditions générales de la licence souscrite par votre établissement. Toute autre reproduction ou représentation, en tout ou partie, sous quelque forme et de quelque manière que ce soit, est interdite sauf accord préalable et écrit de l'éditeur, en dehors des cas prévus par la législation en vigueur en France. Il est précisé que son stockage dans une base de données est également interdit. 


\section{Des couvre-feux à Paris en 1958 et 1961 : Une mesure importée d'Algérie pour mieux lutter contre le FLN?}

Sylvie Thenault

Résumé - L'historiographie récente relative aux événements d'octobre 1961 à Paris a présenté le système répressif alors en place comme le résultat de l'importation, par Maurice Papon, des mesures qu'il connut précédemment au Maroc et en Algérie. Cet article a pour objet de nuancer cette thèse, en examinant précisément le processus de décision du couvre-feu, qui avait aussi été décidé une première fois, en 1958. II apparaît alors que la thèse de l'importation néglige deux aspects : le rôle des revendications policières dans ce processus et la traduction concrète du couvre-feu, sur le terrain. Ainsi, le couvrefeu s'inscrit dans le cadre de la gestion des troupes policières par leur hiérarchie et il renforce le repérage et le fichage des migrants de la région parisienne. 
Tك $\begin{aligned} & \text { l est conseillé de la façon la plus pressante aux travailleurs musul- } \\ & \text { mans algériens de s'abstenir de circuler la nuit dans les rues de } \\ & \text { Paris et de la banlieue parisienne, et plus particulièrement de }\end{aligned}$ 20 h 30 à 5 h 30 du matin. » Cette décision annoncée par un communiqué de la préfecture de police de Paris, en date du 5 octobre 1961, allait donner lieu à l'un des événements les plus controversés de l'histoire de la France contemporaine, devenu un enjeu de mémoire réinvesti par le mouvement antiraciste depuis les années 1980. Appelés par le Front de libération nationale (FLN) à braver l'interdit le 17 octobre, en effet, les Algériens furent victimes d'une répression qui, si elle n'est pas chiffrée de façon incontestable, est d'une ampleur démesurée au regard de l'histoire des manifestations métropolitaines ${ }^{1}$. Ce couvre-feu n'était pourtant pas une première : "Il est conseillé de la façon la plus pressante aux travailleurs nord-africains de s'abstenir de circuler la nuit dans les rues de Paris et de la banlieue parisienne et plus particulièrement de 21 h 30 à 5 h 30 du matin ", avait déjà annoncé, le 2 septembre 1958, un communiqué de la préfecture de police de Paris. Ce premier couvre-feu était simplement tombé en désuétude.

L'historiographie française, pensant les événements parisiens de l'automne 1961 dans le champ clos de la guerre en France, mit face à face police et FLN pour construire un débat autour des responsabilités dans la répression et son ampleur, épinglant tour à tour, pour les opposer, celles du FLN, celles de Maurice Papon et celles de la base policière ${ }^{2}$. Aujourd'hui, ces événements sont revisités à l'aune des leçons tirées des études post-coloniales anglo-saxonnes. Jim House et Neil MacMaster présentent ainsi les violences policières de cette période comme des violences «coloniales » au sens de violences importées des colonies et résultant d'une vision raciste et déshumanisée des " Indigènes ${ }^{3}$ », violences menaçant l'État et la société française d' "algérianisation », pour reprendre leur expression. À leurs yeux, deux critères justifient le recours - inédit dans une historiographie qui n'avait pas, jusque-là, posé la question en ces termes - à l'adjectif « colonial » pour qualifier les mesures de cette période et les violences qui allaient en découler : le contexte géographique et politique de ces mesures, d'abord appliquées dans un Maghreb en ébullition contre la tutelle française où Maurice Papon exerça ; la cible visée, à savoir des sujets coloniaux.

\footnotetext{
1. Tartakowsky (D.), Les manifestations de rue en France, 1918-1968, Paris, Publications de la Sorbonne, 1998. 2. Les deux ouvrages de référence sont ceux de Einaudi (J.-L.), La bataille de Paris, Paris, Seuil, 1991 et de Brunet (J.-P.), Police contre FLN. Le drame d'octobre 1961, Paris, Flammarion, 1999. Sur leur controverse, cf. Thénault (S.), "Le fantasme du secret autour du 17 octobre 1961 », Matériaux pour l'histoire de notre temps, 56, 2000.

3. «The racist and deshumanized view of indigenous people that was deeply entrenched in the colonial order and which spiralled into a vast apparatus of extraordinary brutality during the terminal crisis of decolonization appeared to lie beyond the control of parliament and the judiciary, and threatened to pass like a virus over the Méditerranean and corrupt a "pure" Republican order that was perceived as the guardian of universal human rights and the traditions of 1789. » (House (J.), MacMaster (N.), Algerians, State Terror and Memory, Oxford-New York, Oxford University Press, 2006, p. 27). Livre traduit aux éditions Tallandier sous le titre Paris 1961. Les Algériens, la terreur d’État et la mémoire.
} 
Creusant le sillon tracé par Linda Amiri qui qualifiait, quant à elle, le système de répression parisien de "système Papon ${ }^{4}$ ", devant tout à l'homme et plus précisément à l'exercice de ses fonctions antérieures en terre constantinoise, Jim House et Neil MacMaster insistent en effet particulièrement sur l'expérience de la répression de mouvements terroristes et insurrectionnels acquise par Maurice Papon au Maroc puis en Algérie, d'où il arrive au printemps 1958, lorsqu'il est appelé à la préfecture de police de Paris. Dans un tel contexte historiographique, la reconstitution du processus de décision du couvre-feu en 1961 offre l'opportunité de questionner cette idée majeure d'une importation de «techniques coloniales ${ }^{5}$ » d'Algérie en France par la personne du préfet de police : la décision d'instaurer un couvre-feu visant spécifiquement les Algériens relève-t-elle d'une transposition en métropole des savoir-faire répressifs de sa carrière maghrébine?

Cette question centrale, toutefois, pose le problème dans un contexte extérieur à la métropole. Les policiers, acteurs de la lutte contre le nationalisme algérien en France, sont tenus à l'écart d'une telle interprétation. Or, ils ont joué un rôle décisif dans l'histoire de ces couvre-feux, tant dans la décision de les proclamer que dans leur application. Ce sont eux, par les usages mêmes qu'ils en ont faits, qui ont donné un sens à ces mesures. Relèvent-elles réellement, alors, d'une réponse au terrorisme du FLN?

\section{8-1961, des communiqués aux portées très différentes}

Dans la nuit du 24 au 25 août 1958, le FLN a lancé une série d'attentats visant notamment des infrastructures pétrolières sur tout le territoire français, alors qu'il ne s'était jusque-là manifesté que par des attentats ponctuels prenant des individus pour cible. Cette nuit aurait pu marquer un tournant dans l'histoire de la guerre en France, si la volonté des nationalistes algériens d'ouvrir en métropole un "second front ${ }^{6}$ " avait pu se maintenir dans la durée, ce qui ne fut pas le cas. Sur le moment, toutefois, cette tentative a été perçue comme un changement remarquable de la «tactique » des « rebelles » qui s'attaquaient "pour la première fois aux installations économiques et aux locaux de police ${ }^{7}$.

4. Amiri (L.), La bataille de France. La guerre d'Algérie en métropole, Paris, Robert Laffont, 2004.

5. L'expression est aussi utilisée par House (J.), MacMaster (N.), Algerians, State Terror and Memory, op. cit.

6. Haroun (A.), La $7^{\text {ème }}$ wilaya, Paris, Seuil, 1986, p. 90-111.

7. Rapport sur « Le problème algérien dans la région parisienne - Activités des services de police - Bilan de la lutte contre le terrorisme au 31 décembre 1958 ", archives de la préfecture de police de Paris (dorénavant: APP), Ha 65 (sous dérogation). 
Le couvre-feu annoncé le 2 septembre est alors relayé par une note de service précisant les conditions dans lesquelles des "attestations ", certifiant la nécessité de circuler pendant les heures interdites, peuvent être délivrées aux "Français musulmans d'Algérie ${ }^{8}$ ». La mesure, pourtant, est sans fondement légal - il leur est seulement «conseillé » de "s'abstenir de circuler la nuit »- et son champ d'application est mal défini, tant dans le temps que dans l'espace. "Plus particulièrement de $21 \mathrm{~h} 30$ à $5 \mathrm{~h} 30$ du matin", précise en effet le communiqué, mais que signifie "plus particulièrement » ? La définition de son champ d'application géographique, quant à elle, ne repose pas sur le découpage administratif : l'expression « les rues de Paris et de la banlieue parisienne » désigne-t-elle le département de la Seine?

L'application de ce couvre-feu reste incertaine. Ali Haroun, alors dirigeant de la Fédération de France du FLN, raconte qu'il est tombé en désuétude du fait de l'ordre donné par son organisation de l'ignorer'. Des documents saisis à l'époque attestent effectivement l'existence de telles instructions ${ }^{10}$. Auraientelles suffi, néanmoins, à mettre le couvre-feu en échec si la préfecture de police avait fait preuve de fermeté dans son application? En réalité, l'institution ellemême ne semble pas avoir travaillé en ce sens. Une note sur «l'expérience de 1958 ", affirmant que la mesure ne devait être en vigueur qu'un mois, dresse ainsi un bilan laconique: "Les commissariats ont délivré à ceux qui étaient dans l'obligation de circuler la nuit une autorisation temporaire. Aucun incident sérieux n'a été enregistré au cours de cette expérience ${ }^{11}$. » En janvier 1959, en outre, la réponse faite à Daniel Mayer, qui demandait la suppression du couvre-feu au nom de la Ligue des droits de l'homme, sème le doute sur la réalité de son application, tout en mettant en valeur son caractère flou : la mesure a été " assouplie dans une très large mesure depuis deux mois environ et en fait les contrôles n'ont plus lieu dans la soirée mais seulement la nuit ${ }^{12} »$. Pour Jim House et Neil MacMaster, la préfecture de police, tout simplement, ne disposait pas de l'appareil policier nécessaire à la mise en pratique du couvre-feu ${ }^{13}$.

En 1958, cependant, ce dernier est inséparable d'une autre mesure de restriction de la circulation des Algériens, mesure d'une toute autre portée et visant leurs déplacements en voiture, scooter ou motocyclette, pour reprendre l'énu-

\footnotetext{
8. Note du 2 septembre 1958, APP, Ha 83.

9. Haroun (A.), La $7^{\text {ème }}$ wilaya, op. cit., p. 365.

10. Note de renseignements sans date ni signature, fondée sur la découverte de documents rue Pouchet, dans le XVII ${ }^{\mathrm{e}}$ arrondissement, APP, Ha 83.

11. Note sans date, APP, Ha 83.

12. Lettre de la Ligue des droits de l'homme du 13 janvier 1959 et réponse du 26 janvier 1959, APP, Ha 83. Sur les interventions de la Ligue des droits de l'homme contre les mesures d'exception frappant les Algériens , cf. Blévis (L.), « De la cause du droit à la cause anticoloniale. Des interventions de la Ligue des droits de l'homme en faveur des "indigènes" algériens pendant l'entre-deux-guerres ", Politix, 62, 2003.

13. House (J.), MacMaster (N.), Algerians, State Terror and Memory, op. cit., p. 73.
} 
mération faite dans les documents ${ }^{14}$. Une ordonnance du 7 octobre 1958 autorise en effet la confiscation "sans indemnité » de "tout moyen de transport utilisé par les personnes dangereuses pour la sécurité et l'ordre publics en raison de l'aide matérielle, directe ou indirecte, qu'elles apportent aux rebelles des départements algériens ${ }^{15}$ ». Cette décision vient légaliser « la mise en fourrière » de «tout véhicule suspect " préconisée par le ministère de l'Intérieur à la miseptembre $1958^{16}$. Concrètement, alors qu'elle vise légalement « toute personne dangereuse pour la sécurité et l'ordre publics», elle se traduit par "l'interpellation des Français musulmans d'Algérie propriétaires, conducteurs ou passagers d'un véhicule motorisé », les "véhicules » étant « gardés en consigne devant le poste pendant cinq jours ${ }^{17} »$. Si la personne interpellée est détenue au Centre d'identification de Vincennes, le véhicule est mis en fourrière; sinon, il doit lui être restitué.

Cette décision aboutit à la nécessité, pour tout Algérien propriétaire d'un véhicule, de se munir d'une autorisation auprès du Service d'assistance technique aux Français musulmans d'Algérie (SAT-FMA) de la préfecture de police ${ }^{18}$. Or, la combinaison de cette pièce avec l'attestation nécessaire pour circuler la nuit pose problème : cette attestation va-t-elle jusqu'à autoriser le fait de circuler en voiture? Et inversement : les titulaires d'une autorisation de circuler en voiture doivent-ils, lorsqu'ils roulent de nuit, posséder également l'attestation nécessaire pour se déplacer pendant les heures de couvre-feu ? La réponse de la préfecture de police, rendue en février 1959, alors même que le couvre-feu ne serait plus appliqué, tranche en allant au plus simple : les attestations permettant de sortir pendant les heures de couvre-feu ne visent que les déplacements à pied et les propriétaires de véhicule qui possèdent une autorisation peuvent rouler de jour comme de nuit. Pour théorique que ce raisonnement puisse paraître, surtout que la réponse est rendue tardivement - mais que se passait-il sur le terrain ? - la question n'en avait pas moins une réelle importance pour les commerçants, chauffeurs de taxis et forains, dont la situation avait suscité la note interpellant la préfecture de police sur la combinaison des deux mesures ${ }^{19}$.

Le contrôle de la circulation automobile est resté en vigueur jusqu'à la fin de la guerre. Il a donné lieu à quantité de circulaires, notes et instructions précisant

14. Par exemple dans une note du directeur général de la police municipale en date du 28 septembre 1958, APP, Ha 83.

15. Ordonnance $n^{\circ} 58-916$, article 4 .

16. Texte démarqué d'un message chiffré, adressé par le ministère de l'Intérieur aux IGAMES et préfets de métropole, 16 septembre 1958, APP, Ha 83.

17. Instruction du directeur général de la police municipale, relative à l'application de l'ordonnance, 22 janvier 1959, APP, Ha 83.

18. Sur les SAT-FMA, cf. Amiri (L.), La bataille de France..., op. cit. et Spire (A.), Étrangers à la carte. L'administration de l'immigration en France (1945-1975), Paris, Grasset, 2005, p. 202-205.

19. Note adressée au cabinet du préfet le 31 janvier 1959 et réponse du 23 février, APP, Ha 83. 
les modalités de son application, tout en étant nié chaque fois qu'il a été dénoncé par la presse, par des protestations individuelles adressées aux autorités ou même en justice ${ }^{20}$. Quant au couvre-feu, une note indique qu'il a été réactivé le 11 avril 1959, sans plus de précision : " En raison des circonstances » (lesquelles ?), « appliquer à nouveau, à partir de ce jour et jusqu'à nouvel ordre, les dispositions déjà prises en septembre 1958 et relatives à la circulation (à pied et par tout autre moyen) des Français musulmans d'Algérie à Paris et dans la banlieue entre 22 heures et 5 heures $^{21}$. »

Le 5 octobre 1961, outre le couvre-feu, le communiqué annonce la fermeture des « débits de boissons tenus et fréquentés par des Français musulmans d'Algérie » à 19 heures Il est aussi «très vivement recommandé aux Français musulmans de circuler isolément, les petits groupes risquant de paraître suspects aux rondes et aux patrouilles de police » et ce, de jour comme de nuit. En effet, explique le communiqué, « les attentats sont la plupart du temps le fait de groupes de trois ou quatre hommes ». Cette prescription avait été envisagée en 1958, mais elle avait été retirée du projet, à la demande du directeur adjoint de la police judiciaire ${ }^{22}$.

Les décisions rendues publiques ce 5 octobre -couvre-feu, fermeture nocturne des débits de boissons et interdiction de circuler en petit groupesont aussi annoncées par un « ordre du jour » du préfet de police, transmis par une note de service à l'ensemble de ses subordonnés. L' «ordre du jour » fait office de communiqué à usage strictement interne, dont la confidentialité permet d'ajouter aux mesures rendues publiques l'interdiction de la circulation automobile : «Tout Français musulman circulant en voiture doit être interpellé et, en attendant la décision du commissaire de police ou du Service de coordination des affaires algériennes, la voiture sera mise provisoirement en fourrière ${ }^{23}$. » Le contrôle est devenu interdiction, les autorisations ne semblant plus valoir protection. De toutes ces décisions, la fermeture des débits de boissons est la seule légalisée par une ordonnance du préfet de police, fondée sur l'état d'urgence alors en vigueur en France ${ }^{24}$. Le texte de l'ordonnance diffère cependant de celui du communiqué : les débits de boissons concernés ne doivent pas fermer à 19 heures mais de 20 h 30 à 7 heures du matin. Le fondement légal des mesures reste donc inconsistant et leur arbitraire se niche au cour des variations mêmes de leur présentation, entre leur annonce publique

\footnotetext{
20. La préfecture de police et/ou le ministère de l'Intérieur démentent ainsi un article paru dans L'Humanité le 15 août 1959, nient puis laissent sans suite les réclamations adressées par des sénateurs d'Algérie en janvier 1961 et obtiennent le rejet d'un recours devant le tribunal administratif de Paris le 13 avril 1961 (APP, Ha 83).

21. Note du 11 avril 1959, APP, Ha 83. Deux autres documents, relatifs aux chauffeurs de taxi, témoignent de cette réactivation ( $\mathrm{cf}$. infra).

22. D'après un projet du communiqué, APP, Ha 83.

23. « Ordre du jour » de Maurice Papon en date du 5 octobre 1961, APP, Ha 110.

24. Ordonnance du préfet de police en date du 5 octobre 1961, APP, Ha 110.
} 
par le communiqué, leur transmission en interne par l'ordre du jour et leur contenu légal, quand il existe.

L'application du couvre-feu, cette fois, est attestée de deux manières. D'une part, il est régulièrement l'objet de modifications horaires, prouvant son actualité et ce, jusqu'au cessez-le-feu du 19 mars 1962, impliquant la cessation des "mesures de restriction de la circulation nocturne des Français musulmans d'Algérie ainsi que celles ayant pour objet le contrôle de leurs véhicules ${ }^{25}$ ». Entre-temps, les horaires avaient changé le 3 novembre et le 29 décembre 1961, le couvre-feu ayant été reporté à 22 heures et la fermeture des débits de boissons de 22 heures à 7 heures du matin puis de 22 heures à 6 heures du matin ${ }^{26}$.

D'autre part, l'application du couvre-feu est attestée par le fait qu'il pose des problèmes concrets et nécessite des ajustements sources d'une production documentaire abondante, au contraire du couvre-feu de 1958 qui a laissé peu de traces dans les archives de la préfecture de police. En effet, en 1961 comme en 1958, il est prévu que « tous les travailleurs algériens ayant un emploi qui les conduit à se déplacer la nuit doivent s'adresser aux officiers chefs d'assistance technique aux Français musulmans d'Algérie qui, sur justification, leur délivrent un laissez-passer ${ }^{27} »$. Mais, sous la pression de diverses réclamations, la préfecture est contrainte d'exonérer collectivement des catégories entières de populations. Dès le 6 octobre, le lendemain de l'annonce du couvre-feu, la RATP demande - et obtient - que les cartes de service de son personnel «français musulman d'Algérie » aient valeur de sauf-conduit ${ }^{28}$. Puis, le 17 octobre 1961, le préfet de police doit assurer le président de l'Assemblée nationale que sa mesure ne vise pas les «élus musulmans ${ }^{29}$ ». Le 8 novembre, c'est le service des eaux et nettoiement de la préfecture de la Seine qui saisit le préfet de police $^{30}$. Finalement, le 14 novembre, il est établi que le couvre-feu ne s'applique pas «aux élus Français musulmans d'Algérie, aux hauts fonctionnaires sur présentation de leur carte professionnelle (conseil d'État, cour des comptes, corps préfectoral, administrateurs civils des ministères, etc.), aux agents de la RATP sur présentation de leur carte de service, aux facteurs en tenue d'uniforme, aux personnels de l'assistance publique sur présentation de leur carte professionnelle, aux Marocains et Tunisiens sous réserve de la vérification de leur titre de nationalité, aux étudiants français musulmans d'Algérie sur présentation de leur carte d'étudiant et après contrôle de leur activité, aux employés

25. Note du cabinet du préfet de police, sans date, APP, Ha 110.

26. Notes et ordonnances du préfet de police, APP, Ha 16.

27. Lettre du chef du Service de coordination des affaires algériennes, 27 octobre 1961, APP, Ha 110.

28. Note indiquant que le chef de cabinet du directeur de la RATP a téléphoné à la préfecture de police à ce sujet, le 6 octobre 1961, APP, Ha 110.

29. Lettre du préfet de police au président de l'Assemblée nationale, 17 octobre 1961, APP, Ha 110.

30. D'après une note du 8 novembre 1961, APP, Ha 110. 
des eaux et du nettoiement de la préfecture de la Seine, sur présentation d'une attestation délivrée par leurs ingénieurs, chefs de services ${ }^{31} »$.

En dehors de ces catégories, au 15 novembre 1961, 14000 attestations individuelles ont été délivrées ${ }^{32}$. "Aucun refus n’a été opposé aux demandes", précise une note " au sujet des restrictions de circulation de nuit. » La préfecture évaluant le nombre des « travailleurs algériens » dans le département de la Seine à 90000 , ce serait près du sixième d'entre eux qui a été affranchi du couvre-feu. Renversant cette perspective qui permet d'apprécier positivement la délivrance d'attestations, Jim House et Neil McMaster, quant à eux, soulignent que ce n'est pas le moindre effet du couvre-feu que d'avoir conduit 14000 Algériens dans les locaux des SAT-FMA ${ }^{33}$. La mesure servit ainsi les desseins policiers de repérage et de fichage de ces migrants.

Apparaît ainsi une distorsion entre le motif proclamé de toutes les mesures de restriction de la circulation des Algériens, qu'il s'agisse des couvre-feux ou de leur circulation automobile, et leur usage, sur le terrain. Au nom de la lutte contre le terrorisme, c'est l'entreprise de contrôle des Algériens vivant en région parisienne qui en sort renforcée. La fragilité légale et réglementaire des mesures, sans compter les variations mêmes de leur contenu entre leur annonce publique et leur transmission aux agents chargés de les appliquer, accentue encore le pouvoir dont les policiers jouissent déjà, d'ordinaire, lorsque leurs actes sont encadrés, dans la mise en œuvre des décisions d'ordre public ${ }^{34}$. Ici, en outre, s'ajoute la difficulté à désigner la catégorie visée en recourant aux catégories juridiques classant les populations vivant sur le sol métropolitain et distinguant les nationaux français des autres.

\section{Les « Français musulmans d'Algérie » comme cible}

En tant que mesure discriminatoire visant les Algériens, le couvre-feu révèle le problème posé aux autorités par le statut de ces migrants titulaires d'une " citoyenneté paradoxale ${ }^{35}$ ", qui bouscule la catégorisation binaire fondée sur le critère de l'appartenance à la nation, distinguant les Français d'une part et les étrangers d'autre part. Il s'agissait en effet de savoir comment désigner la catégorie visée, ces « Français musulmans d'Algérie », tout en masquant autant que

\footnotetext{
31. Circulaire du 14 novembre 1961, APP, Ha 110.

32. Note « au sujet des restrictions de circulation de nuit », 15 novembre 1961, APP, Ha 110.

33. House (J.), MacMaster (N.), Algerians, State Terror and Memory, op. cit., p. 100.

34. À l'identique des enseignants, des juristes et des travailleurs sociaux étudiés par Lipsky (M.), StreetLevel Bureaucracy. Dilemnas of the Individual in Public Services, New York, Russel Sage Foundation, 1980.

35. Spire (A.), «Semblables et pourtant différents. La citoyenneté paradoxale des "Français musulmans d'Algérie" en métropole », Genèses, 53, 2003.
} 
possible le caractère discriminatoire des mesures qui suscite maintes protestations $^{36}$. Maurice Papon lui-même l'avait invoqué dans une lettre au Syndicat général de la police (SGP), à propos du contrôle de la circulation automobile des Algériens que le syndicat demandait : « Ce problème revêt un caractère délicat qui ne vous échappera pas en raison de son aspect discriminatoire qui laisserait supposer qu'il y a deux catégories de Français ${ }^{37}$. »

La rédaction même des communiqués annonçant les couvre-feux en témoigne. Outre l'expression de "Français musulmans d'Algérie », la plus utilisée et la plus claire mais qui présente l'inconvénient de souligner qu'il s'agit d'une catégorie particulière au sein de la communauté nationale française, le communiqué du 2 septembre 1958 recourt à celle de «travailleurs nord-africains» et celui du 5 octobre 1961 à celle de "travailleurs musulmans algériens". Les versions successives de la rédaction du communiqué de 1961 témoignent cependant d'une attention et d'une difficulté particulières à exprimer le champ des individus concernés. Dans un premier temps, en effet, «travailleurs nordafricains » avait été écrit, comme en 1958, avant d'être corrigé en «travailleurs algériens » puis, finalement, en "travailleurs musulmans algériens ${ }^{38} »$. La formulation «travailleurs algériens » mettait trop directement en avant l'appartenance à une communauté se définissant sur un autre critère que son statut personnel. Le choix fait d'emblée de privilégier le terme "travailleurs » interroge. S'agissait-il d'une anticipation sur les exonérations qu'il faudrait accepter à l'égard des élus, fonctionnaires, étudiant, etc., que le mot «travailleurs » ne désigne pas d'évidence? Au total, l'élaboration de la rédaction du communiqué de 1961 répond à l'impératif de ne pas reconnaitre les Algériens pour ce qu'ils sont - une communauté nationale - et fonde la définition de la catégorie visée sur un double critère : statut personnel et situation sociale, cette dernière faisant écho au traitement des Algériens comme des «indésirables» dans l'avantguerre d'Algérie ${ }^{39}$. L'adéquation entre terroristes et nationalistes est en revanche remarquable, puisque le qualificatif d' «algériens » est appliqué sans difficulté aux « terroristes » dans la première phrase du communiqué du 5 octobre 1961. Les mesures annoncées auraient pour but « de mettre un terme sans délai aux agissements criminels des terroristes algériens ».

L'expression "travailleurs nord-africains", quant à elle, semble avoir été écartée en 1961 pour éviter d'inclure les Marocains et les Tunisiens. C'est que les réactions de leurs autorités nationales sont attendues et redoutées. Dès le

\footnotetext{
36. Cf. les articles de presse et les protestations conservées dans les archives de la préfecture de police en Ha 83 et en Ha 110.

37. Lettre du 29 août 1958, APP, Ha 89 (sous dérogation).

38. Projet de communiqué, APP, Ha 110.

39. À ce sujet, cf. Blanchard (E.), « La police des “indésirables” : le cas des Algériens de Paris (1944-1954) », Crime, histoire et sociétés, 11 (1), 2007.
} 
mois de septembre, les consuls des deux pays ont protesté contre le traitement de leurs ressortissants lors des opérations de police et, pour le directeur général de la police municipale, « il faut s'attendre à des représentations par voie diplomatique, où on ne manquera pas de faire allusion, par réciprocité, à la sécurité de nos compatriotes résidant au Maroc ou en Tunisie ${ }^{40} »$. Le 12 octobre, Maurice Papon doit également préciser au ministère des Affaires étrangères, saisi par l'ambassadeur du Maroc, qu'il n'existe «aucune équivoque » au sujet du «champ d'application» du couvre-feu, que les Marocains et les Tunisiens «peuvent circuler librement, sous réserve, bien entendu, de la vérification de leur titre de nationalitét ${ }^{41} »$. Puis il reçoit une réclamation marocaine à propos de la fermeture des débits de boissons «tenus et fréquentés par des Français musulmans d'Algérie ». Maurice Papon demande alors au directeur général de la police municipale de « donner toutes instructions aux commissaires d'arrondissement afin d'éviter des difficultés avec les ambassades du Maroc et de Tunisie », tout en précisant que les établissements tenus par des ressortissants des deux pays devront continuer à «faire l'objet d'une surveillance particulière $^{42} »$. Le 31 octobre, enfin, Maurice Papon rappelle par note de service que «sauf dans le cas où leur participation ou leur complicité dans les désordres créés par le FLN serait avérée, tous les citoyens marocains ou tunisiens ne sauraient en aucune façon être appréhendés et conduits dans des commissariats ou dans des centres d'identification. Il ne saurait davantage être porté atteinte à leur intégrité physique ou à leurs biens ${ }^{43}{ }$.

La seule existence de tels ordres prouve que, sur le terrain, Marocains et Tunisiens ont subi les répercussions des décisions de la préfecture de police, du fait de l'unique critère déclenchant les contrôles de police - le faciès - mais aussi du fait de leur solidarité, réelle ou supposée, avec les Algériens. La protection que leur offrent leurs autorités consulaires, ainsi que la pression créée par la présence de ressortissants français dans leurs pays, peut donc paraître toute relative. Il n'en reste pas moins qu'ils sont collectivement moins démunis que les Algériens, soumis par leur statut de migrants coloniaux aux seules autorités françaises, même si des élus locaux d'Algérie dénonçaient à l'Assemblée «les mesures discriminatoires et vexatoires qui frappent aveuglément les pauvres travailleurs sans, pour autant, mettre un terme aux crimes du FLN » ${ }^{44}$.

\footnotetext{
40. Note du directeur général de la police municipale, 20 septembre 1961, APP, Ha 110.

41. Lettre au ministre des Affaires étrangères, 12 octobre 1961, APP, Ha 110.

42. Note pour le directeur général de la police municipale, 18 octobre 1961, APP, HA 110.

43. Note de service du 31 octobre 1961, APP, HA 110.

44. Intervention d'Ahmed Djebbour, député RNUNR (Algérie française) d'Alger, le 6 octobre à l'Assemblée nationale. Sur la protection relative des Algériens par la médiation de représentants politiques, cf. Blanchard (E.), « La police des “indésirables”... », art. cité.
} 
L'exclusion des Marocains et Tunisiens du champ d'application des mesures décidées le 5 octobre 1961, explicitée dans le texte du 14 novembre 1961 précisant la liste des catégories de populations exonérées d'office du couvre-feu ${ }^{45}$, montre que la détermination du groupe visé procède aussi d'une définition par défaut, consistant à préciser qui n'est pas concerné et traçant progressivement les contours de la collectivité ciblée. Il en est de même pour le contrôle de la circulation automobile et ce d'autant plus que le texte même de l'ordonnance le légalisant est détourné. En effet, alors que celle-ci permet la « confiscation » des véhicules de "toute personne dangereuse pour la sécurité et l'ordre publics ", cette désignation, exempte de tout critère de nationalité ou de statut mais fondée sur la dangerosité des personnes, est convertie en «Français musulmans d'Algérie » dans les circulaires, instructions et notes du ministère de l'Intérieur et de la préfecture de police. Cette conversion opère un double détournement du texte légal, en introduisant le critère du statut personnel et en assimilant tout " Français musulman d'Algérie » à un individu potentiellement dangereux. Sur le terrain, le faciès reste d'évidence déterminant, comme en témoigne le cas d'un Français d'Algérie, qui, conduit au Centre d'identification de Vincennes après la confiscation de sa voiture, «se plaint d'avoir été traité comme un Français musulman d'Algérie » et sollicite « une attestation lui permettant de circuler en voiture sans avoir à craindre des ennuis ultérieurs ». La préfecture de police botte en touche : « Il faut donc convoquer M. Aknoun au commissariat et l'informer verbalement que puisqu'il n'est pas Français musulman d'Algérie, il peut circuler en voiture sans autorisation préalable ${ }^{46}$. »

De même, posent problème les «Français d'Algérie d'ascendance musulmane mais relevant du statut civil français pour des raisons diverses (naturalisation avant l'accès général à la citoyenneté française, conversion au catholicisme ou au protestantisme, naissance en France de père musulman et de mère de souche française... $)^{47} \gg$. Alors que le chef du SAT-FMA estime que l'ordonnance du 7 octobre 1958 ne devrait pas leur être appliquée, le cabinet du préfet estime au contraire que «les critères telles (sic) que l'origine ou la religion constituent des éléments d'appréciation qui ne peuvent pas toujours être pris en considération sur le champ, c'est-à-dire sans vérification minutieuse quant à l'activité des intéressés ${ }^{48}$ ». Autrement dit, leur origine française "de souche » ou leur renoncement à la foi musulmane ne les exonère pas du contrôle de leur véhicule. C'est donc, au fond, leur «ascendance musulmane» qui les rend

45. Cf. supra, note 21.

46. Note manuscrite du 3 novembre 1959, APP, Ha 83. Sur ces "Français d'Algérie d'ascendance musulmane ", cf. Blévis (L.), "La citoyenneté française au miroir de la colonisation : étude des demandes de naturalisation des “sujets français” en Algérie coloniale ”, Genèses 53, 2003.

47. Lettre du cabinet du préfet au directeur de la police judiciaire, transmise au chef du SAT-FMA, 28 novembre 1960, APP, Ha 83.

48. Note au chef du SAT-FMA, 28 novembre 1960, Ha 83. 
suspects. C'est cette ascendance que désigne la catégorie «Français musulman d'Algérie » et c'est la traduction de cette ascendance dans l'apparence physique qui expose au contrôle policier.

Ce dernier se concrétise, pour les Algériens, par un harcèlement dont rend notamment compte le nombre d'entrées au Centre d'identification de Vincennes pour la seule année 1960 : 67 281, dont 63392 ont donné lieu à des remises en liberté sans autre forme de procès ${ }^{49}$. Le contrôle de la circulation automobile, quant à lui, est permanent à partir de septembre 1958. Il met directement en évidence les conséquences économiques des mesures répressives subies par les Algériens. Pratiquement, il met au chômage chauffeurs, livreurs, dépanneurs, démarcheurs et exclut les Algériens de ces professions ${ }^{50}$. Lors de la résurgence du couvre-feu au printemps 1959, ce sont les chauffeurs de taxi qui sont visés. Le 29 avril 1959, un conseiller technique du cabinet du préfet de police s'adresse en effet au directeur de la circulation, des transports et du commerce pour l'informer de la décision «d'interdire aux chauffeurs de taxi français musulmans d'Algérie de circuler la nuit » et d'étudier les moyens de légaliser cette décision. À défaut, il souhaite «adresser des recommandations expresses aux intéressés en vue de les dissuader de se livrer, de nuit, à leur activité professionnelle en soulignant les conséquences qui ne manqueraient pas de résulter pour eux de l'inobservation de cette recommandation (notamment conduite dans les centres de triage des Français musulmans d'Algérie en cas de contrôle sur la voie publique $)^{51} »$. Une lettre en ce sens est effectivement envoyée cinq jours plus tard à quatre-vingt-sept artisans algériens, trois syndicats d'employeurs et six employeurs de chauffeurs de taxi ${ }^{52}$.

L'usage des mesures de contrôle de la circulation des Algériens en région parisienne les éloigne de leur objectif proclamé : anéantir le terrorisme du FLN. Cet effet d'éloignement peut être considéré comme le résultat de la traduction pratique de ces mesures par la base policière, sur le terrain, dans ses actes quotidiens. Il apparaît ainsi que la lutte contre le terrorisme ne pouvait être qu'un but indirectement visé par les mesures : s'agissait-il vraiment de gêner les déplacements de la masse pour gêner ceux des seuls groupes armés ? Et si le lien entre lutte contre le terrorisme et contrôle des déplacements des Algériens devient ténu, que dire de la thèse de leur importation par un préfet de police fort de son expérience en terre maghrébine?

49. Amiri (L.), La bataille de France..., op. cit., p. 88. Ce nombre ne signifie pas que 67281 Algériens ont été détenus au Centre d'identification de Vincennes, une même personne pouvant y être conduite plusieurs fois.

50. Cf. la lettre du chef du bureau de renseignements spécialisés (BRS), bureau rattaché au SAT-FMA se plaignant de ces mesures, 14 avril 1959, APP, Ha 83.

51. Note conservée aux APP, Ha 83.

52. C'est le directeur de la circulation, des transports et du commerce qui l'envoie, APP, Ha 83. 


\section{De la lutte contre le terrorisme à la gestion de la base policière}

Une récapitulation générale des attentats commis par le FLN, au 31 décembre 1961, donne un total de 2153 , ayant fait 1391 morts et 1593 blessés $^{53}$. Ce sont les Algériens qui en sont les premières victimes, avec 1269 morts et 1296 blessés, devant les « civils » au nombre de 75 tués et 155 blessés, les policiers ayant compté 47 morts et 142 blessés dans leurs rangs. Un tel bilan, toutefois, éclaire-t-il l'adoption des couvre-feux ? En 1958, en effet, ce n'est pas le nombre de victimes mais le changement de nature des cibles qui est remarqué. C'est bien le fait que le FLN se soit attaqué à des « installations économiques et des locaux de police ${ }^{54}$ ", et non une recrudescence des attentats, qui motive un effort dans la lutte contre le terrorisme. En 1961, la situation diffère. D'après une note sur " les attentats perpétrés contre les forces de l'ordre dans le département de la Seine ", avant l'été 1961, seuls quatre attentats, commis le 25 août 1958, le 18 septembre 1958, le 6 novembre 1959 et le 9 avril 1960, relevaient d'un «terrorisme aveugle ${ }^{55}$ ». Mais depuis le 28 août 1961, « les forces de police ont perdu sept de leurs membres" dont trois dans des attentats au "caractère aveugle». En 1961, le couvre-feu fait suite à la fois à une recrudescence du terrorisme contre les policiers et à l'affirmation de son arbitraire.

Cette mise en relation des attentats du FLN et des décisions de couvre-feu repose sur l'idée que ces décisions, précisément, y répondraient. Or, le couvrefeu n'est jamais cité parmi les moyens de les combattre, y compris dans des documents qui étudient cette question dans les périodes proches de sa proclamation, à un moment où il serait susceptible d'être mis en avant. Mais ce n'est pas le cas. Le rapport du 31 décembre 1958, par exemple, ne dit pas un mot du couvre-feu $^{56}$. Au titre de la lutte contre le terrorisme, il énumère les patrouilles, les "opérations inopinées» de contrôle d'un secteur qui est bouclé, les «battues » après chaque attentat, les enquêtes menées par la Brigade des agressions et violences (BAV), relayées par la création du Service de coordination des affaires algériennes (SCAA), les dispositions légales telles que l'internement, les perquisitions, ou encore la mise en fourrière des véhicules.

En 1961, de même, Maurice Papon adresse le 22 septembre un ordre du jour à ses subordonnés, dans lequel, après leur avoir exprimé son «émotion » face

\footnotetext{
53. APP, Ha 44 (sous dérogation).

54. Rapport sur " Le problème algérien dans la région parisienne », document cité, APP, Ha 65 (sous dérogation).

55. Note du préfet de police pour le ministre d’Etat chargé des Affaires algériennes, 2 novembre 1961, APP, Ha 44 (sous dérogation). Cette note exclut les attentats visant les membres de la force de police auxiliaire, plus connus sous le nom de " harkis».

56. Rapport sur « Le problème algérien dans la région parisienne », document cité, APP, Ha 65 (sous dérogation).
} 
aux attentats, il liste les mesures prises pour les enrayer: reprise de «l'offensive », envoi d'un « convoi hebdomadaire » vers l'Algérie pour éloigner les « oisifs », dotation des policiers d'un gilet pare-balles, protection des commissariats... Il les informe également qu'il a demandé au gouvernement d'adopter trois textes, l'un garantissant l'anonymat des fonctionnaires dans les procédures judiciaires, le deuxième supprimant le sursis dans les peines prononcées pour port et détention d'armes, le troisième modifiant l'ordonnance du 3 juin 1960 relative notamment aux crimes flagrants ${ }^{57}$. Deux semaines avant son adoption, le couvre-feu n'est pas cité.

L'efficacité du seul couvre-feu contre les attentats, par ailleurs, est délicate à évaluer. Cette question, sans objet en 1958 vu le caractère aléatoire de l'application de la mesure, se discute en 1961. Dans une note relative aux « restrictions de circulation de nuit", la préfecture de police se décerne un véritable satisfecit : depuis le couvre-feu du 5 octobre, aucun attentat contre des policiers n'a été commis, le terrorisme contre les Algériens eux-mêmes a nettement régressé et la délinquance de droit commun les impliquant est en recul ${ }^{58}$. Pour autant, est-il possible de mettre de tels résultats uniquement sur le compte des « restrictions de circulation de nuit »? Cela paraît difficile, tant la conjoncture des mois d'octobre et de novembre, marquée par la manifestation du 17 octobre et sa répression, laissait peu de place au terrorisme. Le Comité fédéral du FLN lui-même, en outre, depuis l'été, tentait de reprendre la main sur des groupes armés auxquels il défendait de procéder à des attentats aveugles ${ }^{59}$. Finalement, le décalage est frappant entre, d'une part, la publicité donnée au couvre-feu et, d'autre part, son absence dans les rapports sur les moyens de lutter contre le terrorisme ainsi que son utilité toute relative. Le couvre-feu aurait-il eu pour objet un effet d'annonce? Outre le contexte des attentats du FLN, en effet, les décisions de 1958 et de 1961 font suite à l'expression d'un malaise profond dans les rangs policiers.

Leur mécontentement apparaît d'autant plus comme un facteur décisif qu'en 1958, Maurice Papon a été appelé à Paris après leur fronde du 13 mars. Ce jourlà, un rassemblement dans la cour de la caserne de la Cité a tourné, dans l'excitation, en manifestation devant l'Assemblée nationale où une délégation de représentants a été reçue ${ }^{60}$. Les attentats dont les agents ont été victimes depuis le début de l'année auraient renforcé un sentiment de malaise lié à leur « situation matérielle ${ }^{61} »$. «Nos indices », " nos salaires », scandaient notam-

\footnotetext{
57. Ordre du jour conservé en H1 B1 (sous dérogation).

58. Note « au sujet des restrictions de circulation de nuit », document cité, APP, Ha 110.

59. Les lettres du Comité fédéral à ce sujet ont été publiées par Mohammed Harbi dans la revue Sou'al (7, septembre 1987, p. 71-88).

60. Rapport de synthèse sur la manifestation du 13 mars 1958, APP, Ha 89 (sous dérogation).

61. Communiqué des syndicats de police publié dans Le Monde du 19 février 1958.
} 
ment les manifestants du 13 mars 1958. Ce sont les conditions d'octroi d'une prime de danger, décidée par le gouvernement, qui a mis le feu aux poudres. Maurice Papon arrive ainsi avec pour principale mission de reprendre en main les troupes de la police parisienne, en leur donnant satisfaction et il allait suivre la voie tracée par l'auteur d'une analyse minutieuse de la manifestation du 13 mars. Constatant que «si les marques publiques de mécontentement sont extrêmement rares dans les corps les plus habitués à la discipline, elles prennent l'aspect d'une explosion lorsqu'elles se manifestent », il préconisait de "trouver un moyen de prévention, peut-être sous la forme d'un arbitrage entre les syndicats et l'administration ${ }^{62}$ ». Sans aller jusqu'à un «arbitrage», la leçon du 13 mars 1958 incite le nouveau préfet de police à se montrer à l'écoute des revendications policières. Or, le couvre-feu, sans être une revendication fondamentale, apparait dans les mesures réclamées par les syndicats, portées à la connaissance du ministère de l'Intérieur et de la préfecture de police, au cours de l'année $1958^{63}$. Il est probable, dès lors, surtout dans le contexte de la mise en place d'un nouveau pouvoir, que la mesure ait eu pour but un apaisement de la base policière, même si la chute de la quatrième République, honnie, l'avait déjà, en soi, réconfortée. Le nouveau régime bénéficiait, par contraste, au moment de son avènement, d'un capital de sympathie qu'il s'agissait alors d'entretenir ${ }^{64}$.

Entre 1958 et 1961, les causes du mécontentement se sont déplacées d'une combinaison entre les attentats et la situation matérielle des policiers pour se fixer sur le seul terrorisme du FLN. Maurice Papon, alors, ne cache pas qu'il cherche à calmer ses troupes en accédant, dans la mesure du possible, à leurs vœux. Le 9 octobre 1961, il relate à Roger Frey, ministre de l'Intérieur, qu'il a rencontré "dans les différents services de police», un "état d'esprit dont le développement ne laisse pas de [l]'inquiéter et qui participe d'une certaine lassitude devant l'apparente inutilité des sacrifices consentis». Il invoque la «psychologie «simpliste» du gardien moyen» pour justifier l'attention qu'il porte aux «doléances» formulées par les syndicats demandant le châtiment efficace des « coupables d'assassinats de policiers ${ }^{65}$ ».

Or, en 1961, l'enjeu est encore plus crucial que trois ans plus tôt. C'est l'ombre d'une OAS arrivant en métropole - elle a visé le chef de l'État à Pontsur-Seine le 8 septembre - qui plane sur le pouvoir. C’est son influence au sein

\footnotetext{
62. Rapport de synthèse sur la manifestation du 13 mars 1958, APP, Ha 89 (sous dérogation).

63. Il apparaît pour la première fois dans une lettre de l'Association amicale des gradés de la police municipale, adressée le 10 février 1958 au préfet de police, APP, Ha 89 (sous dérogation). Cf. aussi les motions syndicales conservées en Ha 44 (sous dérogation).

64. Berlière (J.-M.), «Un maintien de l'ordre entre naufrage des principes démocratiques et faillite de l'Etat? ", in Berstein (S.), Milza (P.), Sirinelli (J.-F.), dir., Michel Debré, Premier ministre, 1959-1962, Paris, PUF, 2005.

65. Lettre conservée en Ha 45 (sous dérogation).
} 
des forces de police qu'il s'agit de contrecarrer. Les protestations qui suivent la répression de la manifestation des Algériens le 17 octobre, ainsi, dénoncent en particulier l'influence des policiers de l'ancien réseau Dides, dévoué à la lutte contre le communisme et dissous du fait qu'il constituait une véritable pépinière d'un activisme ancré à l'extrême droite, favorable à l'Algérie française. La police, toutefois, n'avait pas été purgée de ses membres ${ }^{66}$. En 1961, donc, le communiqué annonçant notamment le couvre-feu fait suite à une réunion avec un Comité permanent de coordination et de défense de la police rassemblant les syndicats de la police parisienne. Et cette fois encore, le couvre-feu est décidé pour calmer le jeu. Jim House et Neil MacMaster citant les archives du Syndicat général de la police (SGP), racontent aussi comment, par la suite, Maurice Papon tenta d'obtenir le soutien de cette organisation en argumentant sur le fait qu'il lui avait donné satisfaction en décidant du couvre-feu le 5 octobre 1961 et qu'elle lui devait la réciprocité dans l'échange de services rendus ${ }^{67}$.

Au total, la reconstitution du processus de décision des couvre-feux met en évidence leur émergence par le biais des revendications policières, que le contexte politique - fronde subversive en 1958, OAS à son apogée en 1961 invite à chaque fois à prendre très au sérieux. C'est finalement plus la gestion de ses troupes par le préfet de police qui importe dans la décision que l'existence du terrorisme du FLN en soi. L'expérience de Maurice Papon au Maghreb n'aurait-elle donc joué aucun rôle?

En fait, en 1958, Maurice Papon avait lui-même exprimé un doute sur l'efficacité d'un contrôle de la circulation des Algériens pour lutter contre les attentats, sans que le couvre-feu et le contrôle de la circulation automobile soient clairement distingués. Le ministère de l'Intérieur lui avait demandé son avis sur un certain nombre de mesures réclamées par les syndicats. Mais, alors que le ministère l'interrogeait sur l'opportunité d'une « réglementation spéciale de la circulation nocturne des ressortissants nord-africains », Maurice Papon avait répondu sur leur "circulation automobile», comme si les deux types de mesures s'amalgamaient dans l'idée plus générale d'un contrôle des déplacements des Algériens, une antienne de leur gestion policière sur le sol métropolitain ${ }^{68}$. Maurice Papon se prononçait alors en ces termes : "Il est pratiquement inadmissible de prendre des mesures d'exception concernant la circulation automobile des Nord-Africains. Celles-ci seraient au demeurant parfaitement

66. Gaston Defferre, par exemple, qui a rencontré des responsables syndicaux, réclame une épuration de la police, au Sénat, le 31 octobre : « 80 à $85 \%$ des effectifs de la police parisienne condamnent ces actes [...] mais ils m'ont dit qu'il y avait dans leurs rangs un certain nombre d'hommes prêts à tout. Ils appartenaient à ce que l'on appelait hier le réseau Dides. »

67. House (J.), MacMaster (N.), Algerians, State Terror and Memory, op. cit., p. 148-149.

68. À ce sujet, cf. Blanchard (E.), « La police des “indésirables”... », art. cité. 
inopérantes et n'empêcheraient pas les terroristes décidés à procéder aux attentats. L'enseignement de l'Algérie est à cet effet non négligeable ${ }^{69}$."

Cet avis négatif de Maurice Papon et son fondement sur « l'enseignement de l'Algérie » suggèrent qu'il ne suffit pas de reconstituer la carrière d'agents de l'État pour démontrer un transfert de «techniques » des colonies vers la métropole. Certes, ces agents bénéficient d' « une forme précieuse de capital qui leur permet de revendiquer une compétence spécifique ${ }^{70}$ » pour la gestion de l'immigration et, dans le cas de Maurice Papon, pour la répression d'un mouvement clandestin recourant au terrorisme. Il est indiscutable que la création du SAT-FMA et du SCAA par Maurice Papon à son arrivée à Paris tient à ses affectations antérieures en terre maghrébine, en particulier à Constantine de 1956 à $1958^{71}$. Mais si la reconstitution des carrières est probante en ce qui concerne le cumul de compétences, elle l'est moins sur le terrain du transfert de «techniques » ou de mesures répressives, ce dernier ne résultant pas de la seule circulation des hommes. Au contraire, l'avis négatif exprimé par Maurice Papon montre que le réinvestissement de son passé colonial ne se fait pas sur le mode de la copie conforme, de la simple transposition, de la reproduction à l'identique d'une fonction à une autre, d'un territoire à un autre. Le «travail $\operatorname{archivistique}^{72} »$ prouve que Maurice Papon pense son expérience comme un « enseignement» dont il tire des leçons pour, in fine, préconiser - ou non - des mesures qu'il a vu produire leurs effets dans l'Algérie en guerre et dont il a pu éventuellement constater qu'elles étaient « inopérantes».

Comment expliquer dès lors l'adoption des mesures de 1958 ? Outre le fait que la gestion de l'état d'esprit des forces de police plaidait en leur faveur, il semble qu'en 1958, à la différence de 1961, le ministère de l'Intérieur soit intervenu dans la décision. Une rédaction provisoire du communiqué du 2 septembre mentionnait ainsi que les mesures prises l'étaient «en accord avec l'Intérieur», ce qui, finalement, ne figure pas dans la version rendue publique ${ }^{73}$. La responsabilité du contrôle de la circulation automobile, quant à elle, en toute logique avec l'avis défavorable de Maurice Papon, est renvoyée au ministère de l'Intérieur ${ }^{74}$. Enfin,

69. Avis du préfet de police transmis au ministère de l'Intérieur le 28 août 1958, APP, Ha 89 (sous dérogation). 70. Spire (A.), Étrangers à la carte..., op. cit., p. 190. Françoise de Barros en a fait la démonstration en étudiant le rôle joué en France par les anciens administrateurs des services civils d'Algérie reconvertis en conseillers techniques aux affaires musulmanes (De Barros (F.), "Contours d'un réseau administratif “algérien" ", Politix, 76, 2006.

71. Cf. Amiri (L.), La bataille de France..., op. cit.

72. Pour lequel plaide Sylvain Laurens dans «La noblesse d'Etat à l'épreuve de "l'Algérie" et de l'après $1962 »$, Politix, 76, 2006, p. 76.

73. Projet de communiqué, APP, Ha 83.

74. Il est ainsi noté, au crayon, en marge d'un courrier du chef d'un bureau rattaché au SAT-FMA, le Bureau des renseignements spécialisés (BRS), se plaignant des mesures de restriction de la circulation automobile en raison de leur effet contre-productif sur des Algériens victimes de répression collective : "Ces mesures n'ont-elles pas été imposées par l’Intérieur ? » (14 avril 1959, APP, Ha 83). 
en 1958, la proclamation de couvre-feux dans d'autres départements que celui de la Seine suggère une réflexion à l'échelle nationale, que le ministère de l'Intérieur pourrait avoir assuré. Un couvre-feu est en effet annoncé dans le Rhône et il y en aurait aussi eu un en Seine-et-Oise ${ }^{75}$. L'intervention du ministère de l'Intérieur, en tout cas, caractérise la conjoncture de 1958. La décision auraitelle alors échappé à Maurice Papon? Elle semble bien étrangère, en tout cas, à son expérience en terre coloniale.

\section{Conclusion}

À quelle interprétation des violences policières à l'égard des Algériens de la région parisienne cette histoire des couvre-feux et de leur corollaire, le contrôle de la circulation automobile des Algériens, conduit-elle? Elle tend à nuancer l'idée de leur origine par la seule importation coloniale et à relativiser la qualification du système de répression comme étant un «système Papon ${ }^{76}$ ». Outre le poids de la variable de la gestion du personnel policier dans les décisions et l'intervention de ministère de l'Intérieur, un retour sur la période antérieure accrédite l'idée d'un terreau métropolitain sur lequel Maurice Papon et son expérience coloniale viennent agir. Dès novembre 1957, le directeur d'une société installée dans le XVIII ${ }^{\mathrm{e}}$ arrondissement signalait à la préfecture de police que de nombreux ouvriers arrivaient en retard en raison des contrôles d'identité et demandait que les agents leur remettent un papier « tenant lieu de billet de retard ou justifiant leur absence ${ }^{77} »$. Avant 1954, même, la police procéda, en région parisienne, à de vastes opérations d'arrestations collectives d'Algériens suivies de leur fichage, prenant la forme de «battues » et « rafles » mobilisant "parfois des centaines de policiers dans les quartiers d'habitat algérien $^{78} »$.

La reconstitution de la carrière de Maurice Papon et le pistage des mesures prises à la préfecture de police de Paris sous sa conduite gagneraient à être insérées dans une histoire longue de la gestion policière des Algériens en France pour comprendre par quelles interactions entre terreau métropolitain et importation coloniale le système répressif déployé en région parisienne dans les années 1958-1962 est élaboré - une histoire longue qui devrait restituer, aussi, le rôle des policiers dans ces événements. Ils en furent acteurs en effet, non seulement parce que la répression du nationalisme leur incombait en France, où l'armée n'assuma aucune responsabilité dans la lutte contre le FLN, mais aussi

75. Le 2 septembre 1958, Combat cite un communiqué de la préfecture du Rhône concernant «les rues de l'agglomération lyonnaise ». Cf. aussi Haroun (A.), La $7^{\text {z̀me }}$ wilaya, op. cit., p. 99.

76. L'expression est de Amiri (L.), La bataille de France..., op. cit., p. 81.

77. APP, Ha 83.

78. Blanchard (E.), « La police des “indésirables”... », art. cité. 
parce qu'ils constituaient un corps professionnel doté de sa propre culture et travaillé par ses caractéristiques propres de recrutement, de statut, de rémunération, de syndicalisation, de rapport à la hiérarchie ${ }^{79} \ldots$ Mais l'historiographie, aujourd'hui, sur ce sujet, fait encore défaut.

Sylvie Thénault est chargée de recherche CNRS au Centre d'histoire sociale du $x x^{e}$ siècle (CHS).

sylviethenault@aliceadsI.fr
Parmi ses publications récentes : " L'OAS à Alger en 1962. Histoire d'une violence terroriste et de ses agents ", Les Annales. Histoire, sciences sociales, 5, 2008 ; "L'état d'urgence (1955-2005). De I'Algérie coloniale à la France contemporaine. Destin d'une loi ", Le Mouvement social, 218, janviermars 2007.

79. Alain Dewerpe en explore la culture professionnelle dans Charonne, 8 février 1962. Anthropologie d'un massacre d'État, Paris, Gallimard, 2006. 